\title{
Logistic Constraints on 3D Termite Construction
}

\author{
Dan Ladley and Seth Bullock \\ School of Computing, University of Leeds, UK \\ $\{$ danl, seth\}@comp.leeds.ac.uk
}

\begin{abstract}
The building behaviour of termites has previously been modelled mathematically in two dimensions. However, physical and logistic constraints were not taken into account in these models. Here, we develop and test a three-dimensional agent-based model of this process that places realistic constraints on the diffusion of pheromones, the movement of termites, and the integrity of the architecture that they construct. The following scenarios are modelled: the use of a pheromone template in the construction of a simple royal chamber, the effect of wind on this process, and the construction of covered pathways. We consider the role of the third dimension and the effect of logistic constraints on termite behaviour and, reciprocally, the structures that they create. For instance, when agents find it difficult to reach some elevated or exterior areas of the growing structure, building proceeds at a reduced rate in these areas, ultimately influencing the range of termite-buildable architectures.
\end{abstract}

\section{Introduction}

\section{$1.1 \quad$ Termites}

Termites create some of the most impressive structures seen in nature, building features such as air conditioning, fungus farms and royal chambers into their huge mounds. Recent research has started to explain how these complicated architectures are constructed, but we have yet to fully understand how communities of simple organisms can collaborate successfully on such grand constructions. What seems clear is that the simple termite cannot be relying on centralised control in the form of either a genetic blueprint or guidance from a single executive such as the termite queen. Rather, we now believe that much of the construction process is co-ordinated through stigmergy, a form of indirect communication via environmental cues that are typically produced as a side-effect of the very activity that requires co-ordination [8].

In the case of termite construction work, as building material is assembled, the local environment of the builders is altered in such a way as to encourage appropriate behaviours. Partially built structures "communicate" with workers in such a way as to facilitate their own completion. Over time, as building work continues, the "messages" conveyed by the environment change in subtle ways that allow complicated, heterogeneous architectures to be constructed.

In addition to the physical presence or absence of building material, the environment influences termite building behaviour through supporting various 
diffusive processes. For instance, recently deposited material is a source of a particular kind of "cement" pheromone. This pheromone attracts termites, and thereby encourages the deposition of more building material at or near its source. The consequent positive feedback concentrates building effort in hot-spots, efficiently amplifying what are initially randomly-placed pieces of building material into well-spaced pillars, and subsequently walled enclosures, which may eventually be covered to form chambers. A second pheromone exuded by the queen termite encourages building activity when encountered at a particular concentration level. The pheromone gradient "template" created by a stationary queen encourages material to be deposited at a characteristic distance from her location, eventually leading to the construction of a "royal chamber" found at the centre of many termite mounds. Finally, a third "trail" pheromone, deposited by moving termites, also guides building activity, co-ordinating the formation of galleries and covered walkways. Even wind may affect the structures built by termites, who are thought to be sensitive to air currents and able to make decisions based on direct interaction with the wind. In addition, any wind will disturb pheromone diffusion and influence the structures being built as a result $[6,3]$.

While developing and exploring models of stigmergic, decentralised construction has direct application in insect biology, there also exists the possibility that improving our understanding of these processes might lead to powerful new engineering and design methodologies. Self-assembling robots, automatic repair of space craft or nuclear reactors, and the construction of nanoscale structures are all examples of engineering challenges that might benefit from or even require a termite-inspired approach due to the extreme scales at which the activity must take place, the inaccessibility of the building site, or simply the savings in time and money that could potentially be achieved.

\subsection{Previous Models}

Aspects of the termite mound-construction process have been modelled quite extensively. Deneubourg's mathematical model of pillar formation [4] explained the regular spacing observed in nature as resulting from a positive feedback cycle involving the cement pheromone emitted from recently placed building material. A set of differential equations tracked the movement of termites, diffusion of cement pheromone and location of "active" (recently placed) building material over a two dimensional world. From an initially random distribution of active material "pillars" could form through (i) the amplification of any initial variability and (ii) nearby pillars "competing" for termite attention.

This model was later expanded by Bonabeau et al. [3] to include equations representing factors such as wind, a pheromone template emitted by a stationary queen, and an artificially imposed net flow of ants across the world. The queen pheromone template allowed the structure of royal chambers to be modelled. A net flow of termites led to the construction of walkways and, where these walkways intersected, the formation of what was interpreted as a small chamber. 
Strong winds could prevent any structure from forming, whereas light winds could explain the formation of galleries.

In addition to establishing that complicated structures reminiscent of natural termite mounds can result from very simple rules, these models provide a strong demonstration of the importance of environmental processes in the formation of such mounds. They show that spatio-temporal pheromone properties critically influence construction, and that additional influences such as wind or a net flow of termites can affect building behaviour in interesting ways.

However, these models, and others like them, are limited in certain important respects. First, since they do not explicitly represent the third spatial dimension, they cannot directly represent hollow termite-built structures such as arches, domes, and tunnels. Moreover, they neglect any climbing that termite structures might demand. More importantly, such treatments do not model the logistic constraints on termite movement and pheromone diffusion imposed by the physicality of building material.

In both models described above, the presence or absence of built material has no direct effect on either termite movement or pheromone diffusion. This is important, as it prevents the physical consequences of termite construction behaviour from directly impacting on subsequent termite behaviour, i.e., an important source of stigmergic effects is neglected. For instance, termites may continue to place building material in locations that are physically inaccessible to them, while pheromone may diffuse through solid structures influencing termites that in reality would be ignorant of the pheromone source. Here we label the constraints that these physical realities place on termite behaviour "logistic" since they concern the ability of termites to travel from one particular place to another. While some models of decentralised insect construction have incorporated these constraints, they have not included the role of pheromone diffusion $[2,10$, 1]. In the next section, inspired by a combination of the models mentioned above, we describe a 3-d agent-based model developed in order to explore the impact of logistic constraints on termite construction. Subsequently, we present results from simulations of royal-chamber construction, walkway formation, and the effects of wind. We conclude with a discussion of the role of logistic constraints in decentralised termite construction.

\section{Method}

The simulation comprised a three-dimensional, rectangular lattice $(100 \times 100 \times$ 100) with each location containing diffusing pheromones, and either a simple virtual termite, or solid building material, and, sometimes, wind. The model was updated synchronously in fixed discrete time steps. The lattice is initialised as empty of pheromone and building material and open in all directions, save that the lowest horizontal cross-section contains a single layer of inert building material that represents the ground.

\subsection{Pheromone Behaviour}

The distribution of each type of pheromone changes over time due to the action of three processes: emission, diffusion, and evaporation. 
Emission. Three kinds of pheromone originate from three different kinds of point source. The queen produces a fixed volume of queen pheromone at each time step. Likewise, a trail-following termite produces a constant volume of trail pheromone at each time step. By contrast, the amount of pheromone emitted by each piece of building material is neither constant over time nor unlimited. Rather, each newly placed piece of building material contains an initial finite volume of cement pheromone, a proportion, $0<r \leq 1$, of which is lost to the atmosphere at each time step.

Diffusion. Diffusion between lattice locations that share a common face was modelled as proportional to the pheromone gradient between them using a standard finite volume approach [9]. If the volume of pheromone at a particular location is $x$ and the volume at one of its diffusion neighbours is $y$ then the change of pheromone may be expressed as $\frac{\partial x}{\partial t}=-\alpha(x-y)$, where $0 \leq \alpha \leq \frac{1}{6}$ in order to prevent the creation of pheromone during diffusion. In all results reported here $\alpha=\frac{1}{7}$, which ensures that, at minimum, a pheromone concentration of $\frac{x}{7}$ remains after diffusion, while a maximum of $\frac{x}{7}$ can diffuse to any one diffusion neighbour.

Physical constraints on pheromones are modelled by returning any pheromone that diffuses into a location occupied by building material to its source location. Note that this scheme ensures that a piece of building material that neighbours other pieces on many sides will emit pheromone at a slower rate than a lone piece of building material. Boundary conditions assume that locations beyond the $3-\mathrm{d}$ grid are always empty of pheromone.

Evaporation. At each time step, evaporation was modelled at a rate proportional to the concentration of pheromone at each location by multiplying each concentration by an evaporation constant, $0<v<1$.

\subsection{Termites}

The world contains a fixed number, $n$, of builder termites that remains constant over the course of a simulation. A termite can sense the levels of pheromones at its current location and the pheromone gradients in each direction.

Movement. Termite movement is physically constrained. Of the twenty-six possible adjacent locations available to a termite, it may only move to one that is unoccupied by building material. Additionally, termites may only move to locations that neighbour locations occupied by building material. The first constraint prevents termites moving through walls, while the second constraint forces the termite to move across surfaces, preventing it from flying around the world. If a termite leaves the lattice, which it may do freely, or cannot move to any adjacent location, it is discarded and a new replacement termite is introduced to the lattice.

Builder termites prefer to follow cement pheromone gradients, choosing a new location from the legal alternatives using a roulette-wheel constructed to reflect 
relative gradient strengths. However, with probability inversely proportional to the gradient strength of the selected direction, a random legal move will be made instead. This ensures that, on average, weak gradients exert less influence on termites than strong gradients.

A termite moves $m$ times in this fashion every time step. Since termites move relatively fast by comparison with pheromone diffusion, for all results reported here, $m=5$. Termites are not prevented from entering locations already occupied by other termites. In reality termites are much larger than the pieces of material that they deposit, however, in order to make our model tractable it was necessary to represent termites as single locations. This location was considered to be the head of the termite, i.e. the place it would perform its building and sensing activities. It seems plausible that two termites could be collecting sensory information from the same location at the same time.

Block Placement. For a piece of building material to be placed, the level of queen/trail pheromone at the site must lie within a predefined range $([0.1,0.5]$ for all results presented here) and the site must meet at least one of the following three conditions, which are intended to impose a crude physics on the world:

1. Either the location immediately underneath or immediately above the site must contain material.

2. The site must share a face with a horizontally adjacent location that contains material and satisfies (1)

3. One face of the site must neighbour three horizontally adjacent locations that each contain material.

The first constraint allows vertical stacks to be built. The second allows these stacks to be extended horizontally to a limited degree, while the third allows the gradual construction of elevated horizontal surfaces if sufficient support is present.

If these conditions are met, a block will be placed at the termites current location with probability, $p$, the termite will be removed from the lattice, and a replacement introduced. This is intended to represent the constraint that termites must forage for building material. Notice that once placed, building material cannot be removed or relocated.

Path-Following. In addition to the queen, and builder-termites, a third class was modelled. These termites lay and follow trail pheromone, but are not involved in any building activity. They represent termites who are leaving the nest to forage or returning to the nest having foraged. The model does not allow for foragingtermites or building-termites to change role during the simulation. A group of adjacent, ground-level entry points are specified at the edge of the lattice. At each time step, and with probability, $c$, each of a fixed number of termites may enter the lattice at a randomly chosen entry point. Each trail termite moves across the lattice in a direction roughly perpendicular to the lattice edge at which they enter. However, trail termites are not restricted to move in a straight line. We 
extend an approach employed by Deneubourg [5] to three dimensions in allowing a trail termite to move to any down-stream location that shares a face, edge or corner with its current location and is physically accessible. Trail termites are attracted to trail pheromone in the same way that builder termites are attracted to cement pheromone. If a termite moves off the lattice it is removed.

\subsection{Wind}

Wind was modelled in a deliberately simple manner, originating at a constant fixed strength, $0<s<1$, from one vertical face of the lattice, travelling perpendicular to this face, horizontally across the world, flowing around built structures, and influencing pheromone diffusion. In general, therefore, wind flows from one up-wind vertical cross-section of lattice, $a_{i}$, to the adjacent, down-wind, vertical cross-section, $a_{i+1}$. Wind strength in $a_{i+1}$ can be calculated in the following manner. For every location, $u$ in $a_{i}$, calculate the number of locations in $a_{i+1}$ that share an edge, corner or face with $u$ and are empty of building material. Divide the strength of wind at $u$ equally among these locations in $a_{i+1}$, or (unrealistically) discard the value should no down-stream locations be empty of building material.

In areas lacking building material, this approach will result in wind strength remaining constant as the strength at each location will be distributed evenly across down-wind locations. In areas with obstructions wind strength will increase near exposed surfaces, but be reduced in "sheltered" areas, as the wind flows around the obstruction.

At each location, wind transfers a proportion of pheromone equal to its strength, $s$, to the location immediately down-wind (unless that location is occupied by building material). While wind can thus only carry pheromone in one direction, in combination with diffusion, it is capable of transporting pheromone around structures effectively.

\section{Results}

Simulations were carried out to examine the effects of (i) a pheromone gradient established by a stationary queen, (ii) wind, (iii) a net flow of termites in one direction, and (iv) a net flow of termites in two orthogonal directions.

\subsection{Royal Chamber}

The queen, represented by one or more contiguous blocks in the form of a rough half-cylinder, is placed at the centre of the world in contact with the ground. Each block is a fixed-rate source of queen pheromone. Throughout the simulation the queen remains stationary and is somewhat equivalent to building material in that she obstructs the flow of pheromone, and impedes movement of termites, allowing them to climb over her though not to build on her. 

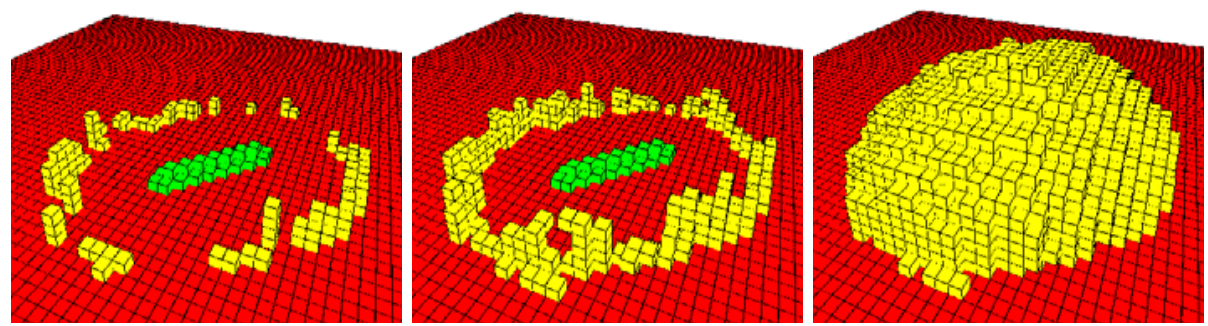

Fig. 1. A royal chamber being constructed. Parameters: $r=0.5, \alpha=\frac{1}{7}, v=0.1, p=$ $0.1, n=300, m=5, s=0.0$
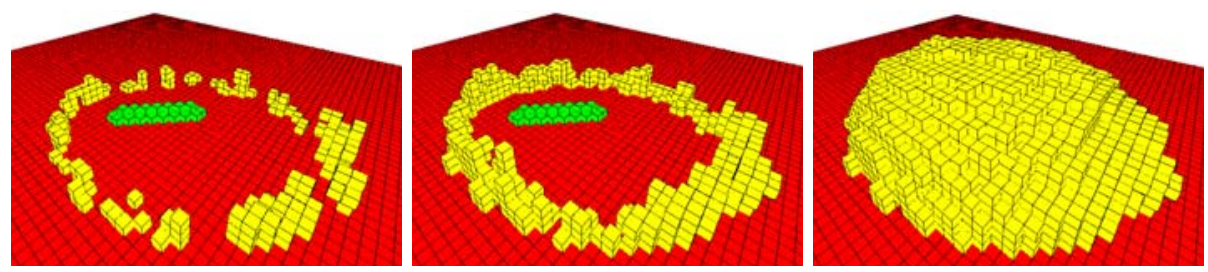

Fig. 2. A royal chamber being constructed under mildly windy conditions (wind emanates from the upper-left lattice edge). Parameters as figure 1, except: $s=0.15$

The simulation is initially run for 1000 time steps without the presence of builder termites to allow the queen's pheromone template to become established. At this point termites are randomly allocated around the outside of the world in contact with the ground, and the model proceeds as described above. Unless otherwise noted the simulations were run with 300 building termites $(n)$, a probability of block placement $(p)$ of 0.1 , a pheromone evaporation rate $(v)$ of 0.1 and a pheromone output rate $(r)$ of 0.5 .

Figures 1 and 2 depict the construction of a royal chamber without wind, and under mildly windy conditions, respectively. First, pillar-like structures are formed at roughly regular spatial intervals, and at a specific distance from the queen. Subsequently, these pillars merge to form a wall that encircles the queen. Finally, the termites achieve a complete dome encompassing the pheromone source. In the absence of any disturbance, termites achieve a roughly hemispherical structure centred on the queen, that may echo the queen's physical shape if she is large enough in relation to the dome (see figure 1). However, in figure 2 the shape of the chamber has been influenced by wind blowing from the upper-left edge of the lattice. As a result, the chamber is not centred on the queen, and is distorted in both it's horizontal and vertical profile, e.g., exhibiting a steeply rising exposed face and a more gradually descending, sheltered, down-stream slope. At higher strengths, wind can prevent a chamber from being completed successfully, or even prevent any construction from becoming established at all. 

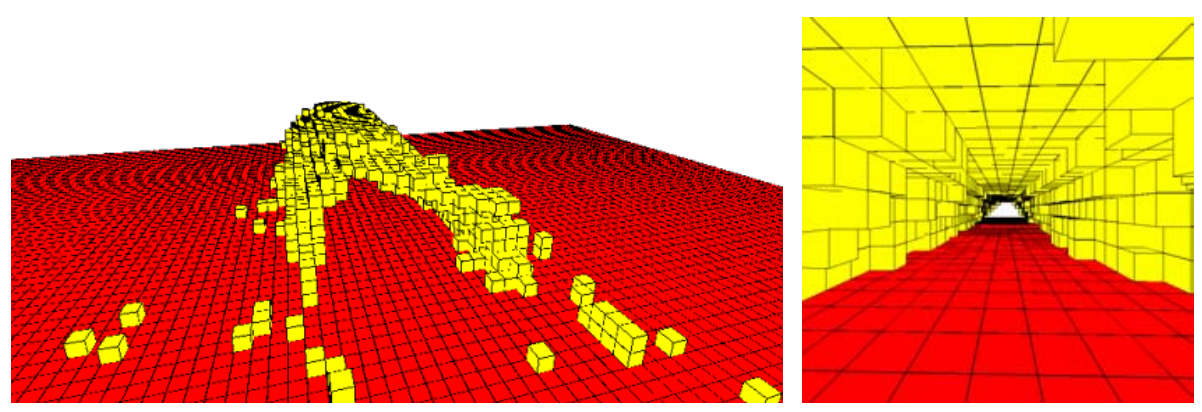

Fig. 3. A covered walkway is constructed. Parameters as figure 1, save that no wind has been modelled, and a flow of trail termites has been introduced: $t=10, c=0.5$. The tunnel's interior is clear of obstructions, and the cross-section is quite regular

\subsection{Covered Walkway}

A narrow flow of termites across the world is introduced by allowing trail termites to enter at ground level from central locations along one lattice edge. Builder termites enter the lattice at the same locations after 1000 time steps. Figure 3 shows the resulting tunnel, partially formed, and an internal view. Construction of the tunnel walls obviously precedes completion of the roof. At the end of the simulation the tunnel is straight, quite regular in cross-section, and clear of obstructions. Notice that, unlike previous models [3] the trail and distribution of trail pheromone are not explicitly defined, but arise from the ongoing activity of trail termites.

\subsection{Crossing Paths}

Two perpendicular flows of termites are introduced, crossing at the centre of the ground plane. Figure 4 depicts the cross-road structures that arise. Despite the potential for interference between trail pheromones from each path, and between the building work at each tunnel (especially given the stochastic rate at which both trails and tunnels are established), each tunnel remains unobstructed internally, and a working covered cross-road junction is achieved.

Figure 4 left depicts a scenario in which builder termites always enter the world from a random location along the ground-level edge of the lattice. The covered walkways that result reliably exhibit a tendency to "mushroom" at their down-stream ends. Constraining the building termites to enter at the same locations employed by the trail termites extinguishes this tendency (figure 4 right). In both cases, however, the interior of the tunnel remains clear of obstructions.

\section{Discussion}

The primary result exhibited by the model is that, pleasingly, the introduction of physical limitations on pheromone diffusion and termite movement have not 

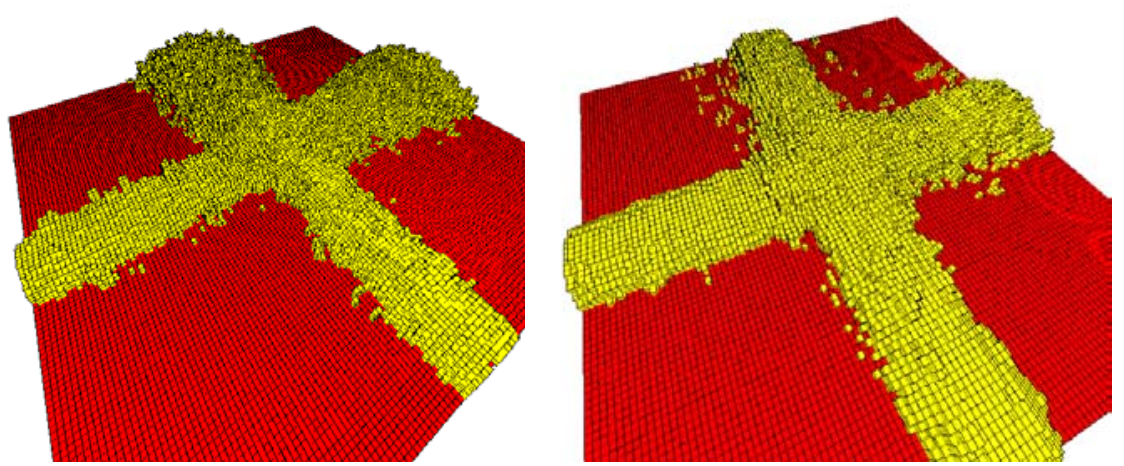

Fig. 4. Two examples of cross-road formation. Parameters again as figure 1, save that no wind is modelled, and two perpendicular streams of trail termites are introduced: $t_{1}=10, t_{2}=10, c=0.5$. At each time step, between zero and 10 builder termites enter the lattice, with probability 0.5 per termite. Left - Builder termites enter from random locations along any edge of the ground plane. Some "mushrooming" of the down-stream sections of tunnel tends to occur. Right - Builders enter from the same entry points employed by trail termites. Mushrooming is suppressed

prevented the building of structures suggested by previous models that neglected such logistic constraints $[4,3]$.

As reported by Deneubourg [4], pillar formation can be driven by simple positive feedback involving a cement pheromone. His results from one- and twodimensional models built on differential equations can be supported by our 3d agent-based model. For instance, in our simulations, pillars form at regular spatial intervals in the early stages of the construction of the royal chamber (see figure 1).

Our model also agrees with the predictions of Bonabeau et al. [3], who showed, in an extension of [4], that the introduction of a source of diffusing queen pheromone can first guide the construction of pillars and subsequently an encircling wall. Our results show that this process can result in a fully-formed 3 -d royal chamber. The manner in which our results are influenced by wind also agrees with previous findings, which reported a distortion of the horizontal crosssection of the royal chamber. However, out treatment of the third dimension also allows us to explore distortion to the elevation of the chamber.

Finally, the Bonabeau model suggested that an artificially imposed flow of termites emitting trail pheromone could result in the construction of walkways. Our simulations extend these results by showing that these structures tend to become covered walkways over time, and remain clear of building material (see figure 3). Previous speculation in [3] that two intersecting trails could give rise to the formation of a cross-road structure are confirmed by our model, which additionally demonstrates that such an intersection will not tend to become blocked by building activity (see figure 4 ).

In addition to confirming work from previous models, our approach has generated new insights. First, and most generally, it is perhaps remarkable that the 
introduction of physical constraints on termite motion and pheromone behaviour have not had more influence on the structures achieved. It was possible, for instance, that as a tunnel was formed and enclosed the trail within it, it would become difficult for trail pheromone to escape, distorting the pheromone distribution in such a way as to interfere catastrophically with new building activity. Moreover, when a piece of building material is placed it cannot be removed in this model meaning that tunnel blockages, should they arise cannot be rectified, making tunnel completion impossible.

In order to implement constrained movement and diffusion we introduced an explicit third dimension. This alone could have introduced dramatic differences between the behaviour observed in our system and in previous models. Climbing, for instance, plays no part in 2-d models, but is important in the current work, since agents must be able to reach a location in order to build there. Despite this, all of the structures suggested by the two relevant previous models were achieved. However, the fact that, in our model, it is harder to reach some locations than others causes agents to build more slowly in hard to reach places like roofs of structures, or the tops of pillars, which require time consuming assents compared to the walls which are at ground level.

Predictable, as the strength of simulated wind increased the building process became slower until a point was reached where no building was possible. While the effect of wind may simply be to disturb a pheromone template to the extent that builders are not encouraged to lay down building material at any location, it also has a more subtle influence on the ability of newly-formed structures to recruit builder termites. Without wind, cement pheromone is free to diffuse in every unobstructed direction, attracting termites to the emitting structure from a wide area. In the presence of wind, however, the cement pheromone cannot diffuse as effectively, hampering the recruitment of up-wind builder termites. This ensures that mildly windy conditions result in differential construction activity.

Rather than being severely limited by the physical obstruction imposed by the results of their own building behaviour, it appears that in some cases these effects can increase the efficiency of the building work and prevent some "problematic" kinds of construction activity such as the tendency for tunnels to "mushroom" observed in figure 4. This "overbuilding" stems from a combination of two factors, (i) a slight "fanning" of the trail laid by the termites due to their stochasticity and amplified by confusion at the intersection of two perpendicular pheromone trails, and (ii) a tendency for builder termites to approach the trail from all sides. These conditions lead to building behaviour near the outer edge of the trail "fan", which is amplified and ultimately results in wide tunnel walls. By contrast, where builder termites enter the lattice at the same points as the trail laying termites, they tend to begin building at a regular distance from the trail mid-line. As a tunnel is formed in this way, it limits the movement of builders, directing them to the tunnel end and preventing them from reaching the outer edge of the "fanned" distribution of trail pheromone. In this way, despite the inability of the trail termites to form a narrow, fixed-width pheromone template, building work is able to produce a narrow, fixed-width tunnel. 


\subsection{Future Work}

Currently, several aspects of the model are under-developed, or unexplored. The three kinds of pheromone employed have not been explored in concert. In the results reported, only two types of pheromone were ever employed together. For instance, it would be interesting to explore the role that trail pheromone deposited by builder termites might play in directing them to sites of recent building activity. How would cement and trail pheromone interact in this situation? For example, it is possible that such an interaction might account for the maintenance of an entrance to the royal chamber, by inhibiting building work across routes employed by many builders. While several entrances might be maintained initially, over time one might expect a single location to dominate and the others to be closed.

Both the crude physics of construction, and the implementation of wind, which currently cannot support swirling eddies or any kind of back-flow, could be improved. The former might benefit from an approach similar to that taken by Funes \& Pollack [7] in their work on simulating Lego structures. Currently, it is possible for unrealistic or impossible structures to be built. A more sophisticated physics might encourage termites to adopt more complicated strategies in order to achieve large-scale structures.

Several logistic factors have yet to be incorporated into our model. Currently, termites do not obstruct one another's movement, and, unrealistically, are assumed to occupy roughly the same amount of space as a piece of building material. For reasons of simplicity, we do not yet explicitly model the tasks involved in discovering raw materials or transporting them to the construction site. The significance of these factors remains to be seen.

More generally, closer comparison between the current model and the detailed behaviour of previous mathematical models is required, and a more extensive characterisation of the our simulation's dependencies on parameter values and initial conditions must be undertaken.

\section{Conclusion}

In conclusion, we have shown that physical and logistic constraints imposed on termite construction by the results of their own building behaviour do not prevent the formation of several structures reminiscent of natural termite architecture. Indeed, it appears that in some cases these constraints can have a positive effect on the ability of termites to achieve efficient, effective constructions.

\section{References}

1. E. Bonabeau, S. Guérin, D. Snyers, P. Kuntz, and G. Theraulaz. Three-dimensional architectures grown by simple 'stigmergic' agents. BioSystems, 56:13-32, 2000.

2. E. Bonabeau, G. Theraulaz, E. Arpin, and E. Sardet. The building behavior of lattice swarms. In R. A. Brooks and P. Maes, editors, Artificial Life IV, pages 307-312. MIT Press, Cambridge, MA, 1994. 
3. E. Bonabeau, G. Theraulaz, J.-L. Deneubourg, N. R. Franks, O. Rafelsberger, J.-L. Joly, and S. Blanco. A model for the emergence of pillars, walls and royal chambers in termite nests. Philosophical transactions of the Royal Society of London, Series B, 353:1561-1576, 1997.

4. J.-L. Deneubourg. Application de l'ordre par fluctuations à la description de certaines étapes de la construction du nid chez les termites. Insectes Sociaux, 24:117130, 1977.

5. J.-L. Deneubourg, S. Goss, N. Franks, and J.-M. Pasteels. The blind leading the blind: Modelling chemically mediated army ant raid patterns. Journal of Insect Behaviour, 2:719-725, 1989.

6. J.-L. Deneubourg, G. Theraulaz, and R. Beckers. Swarm-made architectures. In F. J. Varela and P. Bourgine, editors, First European Conference on Artificial Life, pages 123-133. MIT Press, Cambridge, MA, 1992.

7. P. Funes and J. Pollack. Computer evolution of buildable objects. In P. Husbands and I. Harvey, editors, Fourth European Conference on Artificial Life, pages 358367. MIT press, Cambridge, MA, 1997.

8. P.-. P. Grassé. Termitologia, Tome II - Foundations des societés - construction. Masson, Paris, 1984.

9. C. Hirsch. Numerical Computation of Internal and External Flows, Volume 1: Fundamentals of Numerical Discretization. Wiley, Chichester, 1988.

10. G. Theraulaz and E. Bonabeau. Modelling the collective building of complex architectures in social insects with lattice swarms. Journal of Theoretical Biology, 177:381-400, 1995. 